\title{
Improved method for partial-wave decomposition of two-pion exchange three-nucleon force
}

\author{
Rimantas Lazauskas * \\ IPHC, IN2P3-CNRS/Université Louis Pasteur BP 28, F-67037 Strasbourg Cedex 2, France
}

\begin{abstract}
In this article an efficient method to calculate the matrix elements of three-nucleon force is presented. The new method is improved version of partial-wave decomposition of ref. [1], which simplifies expression to be evaluated and permits to reduce computational effort as much as six times. Proposed method naturally applies to Faddeev-type calculations but also can be used by any method relaying on partial-wave decomposition.
\end{abstract}

\section{INTRODUCTION}

Three nucleon force (3NF) becomes an indispensable ingredient in realistic atomic nucleus calculations. In fact discussion is still vivid about the origin and structure of dominant three-nucleon force terms but even largest sceptics recognize its importance in nuclear physics [2]. Complexity of three-nucleon force turns to be a major obstacle towards its better understanding. Numerical implementation requires enormous analytical and programming effort. At the same time even the best algorithms evaluating 3NF matrix elements become huge computer resources demanding tasks. In ref. 1] a nice Partial Wave Decomposition (PWD) technique of 3NF originating from two-pion exchange diagrams has been demonstrated. The present article improves this PWD by helping to simplify numerical implementation procedure as well as reducing number of necessary floating point operations.

\section{GENERAL REMARKS ABOUT PWD TECHNIQUE}

Three-nucleon force naturally decompose into three parts, which are identical up to a cyclic or anticyclic permutation of the three interacting particles:

$$
V_{i j k}=V_{j k}^{(i)}+V_{k i}^{(j)}+V_{i j}^{(k)}
$$

here in term $V_{i j}^{(k)}$ particle $k$ is considered as a formal spectator. While three-nucleon force $V_{i j k}$ is symmetric for exchange of any two nucleons, term $V_{i j}^{(k)}$ is symmetric only for interchange of particles $i$ and $j$. When working in isospin formalism,- since systems wave function $\Psi$ is fully antisymmetric,- it is necessary to evaluate only one of three terms to get expectation value of the $3 \mathrm{NF}$ acting between three particles:

$$
\left\langle\Psi\left|V_{i j k}\right| \Psi\right\rangle=3\left\langle\Psi\left|V_{i j}^{(k)}\right| \Psi\right\rangle \text {. }
$$

Faddeev-type equations also require evaluation of single 3NF term, if they are written as follows:

$$
F_{i j}^{(k)}=G_{0} V_{i j} \Psi+G_{0} V_{i j}^{(k)} \Psi
$$

here $F_{i j}^{(k)}$ is so called Faddeev component, $V_{i j}$ is two-nucleon interaction potential between nucleons $i$ and $j$. Faddeev components are not fully antisymmetric, however they are antisymmetric for interchange of the nucleons in the active pair (i.e. component $F_{i j}^{(k)}$ is antisymmetric for exchange of nucleons $i$ and $j$ ). Fully antisymmetric systems wave function is a sum of three Faddeev components $\Psi=F_{j k}^{(i)}+F_{k i}^{(j)}+F_{i j}^{(k)}$.

It is useful to work with Jacobi coordinates or momenta. One has three different Jacobi momenta sets, each selecting one of three particles as a spectator. I.e. if we select particle $(k)$ as spectator, associated Jacobi momenta are defined by $\vec{p}_{k}=\frac{1}{2}\left(\vec{k}_{j}-\vec{k}_{i}\right)$ and $\vec{q}_{k}=\left(\frac{2}{3} \vec{k}_{k}-\frac{\vec{k}_{i}+\vec{k}_{j}}{3}\right)$, where $k_{i}, k_{j}$ and $k_{k}$ are individual momenta of the particles. This

*Electronic address: rimantas.lazauskas@ires.in2p3.fr 
momenta set is proper to Fadeev component $F_{i j}^{(k)}$. Following the standard procedure each Faddeev component $F_{i j}^{(k)}$ is expanded in its proper bipolar harmonic basis $|p q \alpha\rangle_{k}$ :

$$
|p q \alpha\rangle_{k} \equiv\left|p_{k} q_{k}\right\rangle\left|\left(l_{p}\left(s_{i} s_{j}\right)_{s_{p}}\right)_{j_{p}}\left(l_{q} s_{k}\right)_{j_{q}}\right\rangle_{J M}\left|\left(t_{i} t_{j}\right)_{t_{p}} t_{k}\right\rangle_{T T_{z}},
$$

here index $\alpha$ spans all the symmetry allowed combinations of the quantum numbers presented in the brackets: $l_{p}$ and $l_{q}$ are the partial angular momenta associated with respective Jacobi momenta; $s_{i}$ and $t_{i}$ are the spins and isospins of the individual nucleons. Three nucleon system conserve total angular momentum $J$ and its projection $M$. Individual isospins of the three nucleons couple to the total isospin $T$ and its magnetic quantum number $T_{z}$.

Separation of the fully symmetric three-nucleon force into symmetric binary terms, as shown in eq.(1), can be done in infinitely many different ways. In practice one associates the binary term $V_{i j}^{(k)}$ with an expression obtained from the Feynman diagram where nucleon $k$ is chosen as a formal spectator. Three-nucleon force related with two-pion exchange diagrams in its general form is usually written as [3]:

$$
\begin{aligned}
V_{i j}^{(k)}= & \frac{\left(\vec{\sigma}_{i} \cdot \vec{Q}_{i}\right)\left(\vec{\sigma}_{j} \cdot \vec{Q}_{j}\right)}{\left(\vec{Q}_{i}^{2}+M_{\pi}^{2}\right)\left(\vec{Q}_{j}^{2}+M_{\pi}^{2}\right)} f_{i} f_{j} \times \\
& {\left[C_{1}\left(\vec{\tau}_{i} \cdot \vec{\tau}_{j}\right)+C_{3}\left(\vec{\tau}_{i} \cdot \vec{\tau}_{j}\right)\left(\vec{q}_{i} \cdot \vec{q}_{j}\right)+C_{4}\left(\vec{\sigma}_{k} \cdot \vec{Q}_{i} \times \vec{Q}_{j}\right)\left(\vec{\tau}_{k} \cdot \vec{\tau}_{i} \times \vec{\tau}_{j}\right)\right] }
\end{aligned}
$$

where $C_{1}, C_{3}$ and $C_{4}$ are simply multiplicative constants and $f_{i}$ is scalar regularization function intended to cut out high-momenta contribution of the nucleon $i$; there is no strict regularization procedure to define cut-off function $f_{i}$ and several different regularization procedures have been used [3, 4, 5].

The last expression can be written in a compact form as:

$$
V_{i j}^{(k)}=C_{1} W_{a}^{(i)} W_{a}^{(j)} I^{(k)}+C_{3}\left(\vec{W}_{b}^{(i)} \cdot \vec{W}_{b}^{(j)}\right) I^{(k)}+C_{4}\left({ }^{(k)} \vec{W}_{d}^{(j)} \cdot \vec{W}_{b}^{(i)}\right) I_{0}
$$

with $I^{(k)}=\left(\vec{\tau}_{i} \cdot \vec{\tau}_{j}\right), I_{0}=\left(\vec{\tau}_{k} \cdot \vec{\tau}_{i} \times \vec{\tau}_{j}\right), W_{a}^{(i)}=\tilde{f}_{i}\left(\vec{\sigma}_{i} \cdot \vec{Q}_{i}\right), \vec{W}_{b}^{(i)}=\widetilde{f}_{i}\left(\vec{\sigma}_{i} \cdot \vec{Q}_{i}\right) \vec{Q}_{i},{ }^{(k)} \vec{W}_{d}^{(j)}=$ $\tilde{f}_{j}\left(\vec{\sigma}_{j} \cdot \vec{Q}_{j}\right)\left[\vec{Q}_{j} \times \vec{\sigma}_{k}\right]$ and $\tilde{f}_{i}=f_{i} /\left(\vec{Q}_{i}^{2}+M_{\pi}^{2}\right)$. The momentum transfer $\vec{Q}_{i}=\vec{k}_{i}^{\prime}-\vec{k}_{i}$ is proper to Jacobi momenta set, where nucleon $i$ is considered as spectator, giving: $\vec{Q}_{i}=\vec{q}_{i}^{\prime}-\vec{q}_{i}$.

In the following we will omit multiplicative constants $C$. Then all three terms of eq.(6) applied on the vector $\Psi$ regain the following form:

$$
\Psi^{\prime} \sim W^{(i)} I W^{(j)} \Psi
$$

Hüber et al. [1] proposed a nice method to evaluate these terms using the following expression:

$$
\begin{aligned}
{ }_{k}\left\langle p^{\prime \prime \prime \prime} q^{\prime \prime \prime \prime} \alpha^{\prime \prime \prime \prime} \mid \Psi^{\prime}\right\rangle= & \sum \sum^{\prime \prime \prime \prime} \sum^{\prime \prime} \frac{(-)^{J-J^{\prime}}}{\widehat{J}}{ }_{k}\left\langle p^{\prime \prime \prime \prime} q^{\prime \prime \prime \prime} \alpha^{\prime \prime \prime \prime} \mid p^{\prime \prime \prime} q^{\prime \prime \prime} \alpha^{\prime \prime \prime}\right\rangle_{j} \\
& \times{ }_{j}\left\langle p^{\prime \prime \prime} q^{\prime \prime \prime} \alpha^{\prime \prime \prime}\left\|W^{(i)}\right\| p^{\prime \prime} q^{\prime \prime} \alpha^{\prime \prime}\right\rangle_{j}{ }_{j}\left\langle p^{\prime \prime} q^{\prime \prime} \alpha^{\prime \prime}|I| p^{\prime} q^{\prime} \alpha^{\prime}\right\rangle_{i} \\
& \times{ }_{i}\left\langle p^{\prime} q^{\prime} \alpha^{\prime}\left\|W^{(j)}\right\| p q \alpha\right\rangle_{i}{ }_{i}\langle p q \alpha \mid \Psi\rangle,
\end{aligned}
$$

here we have introduced convenient abbreviation $\widehat{J} \equiv 2 J+1$. In this expression $J$ is the angular momentum of the three nucleon system and eventually of the bases $\alpha^{\prime \prime \prime \prime}, \alpha^{\prime \prime \prime}$ and $\alpha$. If potential term $W$ is defined as vector $\left(\vec{W}_{b}\right.$ and $\vec{W}_{d}$ cases) the angular momentum $J^{\prime}$ of the states $\alpha^{\prime \prime}$ and $\alpha^{\prime}$ can be different from $J$. Last expression permits to evaluate matrix elements of the potentials $W$ in their natural coordinate set, where $Q$ is independent of one Jacobi momenta ( $p$ or $q$ ). This makes evaluation of matrix element ${ }_{i}\left\langle p^{\prime} q^{\prime} \alpha^{\prime}\left\|W^{(j)}\right\| p q \alpha\right\rangle_{i}$ quite rapid. Nevertheless one requires two Jacobi basis transformation operations, namely ${ }_{k}\left\langle p^{\prime \prime \prime \prime} q^{\prime \prime \prime \prime} \alpha^{\prime \prime \prime \prime} \mid p^{\prime \prime \prime} q^{\prime \prime \prime} \alpha^{\prime \prime \prime}\right\rangle_{j}$ and ${ }_{j}\left\langle p^{\prime \prime} q^{\prime \prime} \alpha^{\prime \prime}|I| p^{\prime} q^{\prime} \alpha^{\prime}\right\rangle_{i}$, which consumes almost all the time required to estimate 3NF terms. Furthermore all the intermediate states $\left(\alpha^{\prime}, \alpha^{\prime \prime}\right.$ or $\left.\alpha^{\prime \prime \prime}\right)$ does not retain antisymmetry with respect to interchange of the nucleons in the active (coupled) pair. Therefore one is obliged to double the size of intermediate bases by including unphysical symmetric states, which are comfortably ignored when using Faddeev-type representation of systems wave-function. 


\section{IMPROVED PWD TECHNIQUE}

In this study we propose to redistribute 3NF terms in Faddeev equation, in the following manner:

$$
\widetilde{F}_{i j}^{(k)}=G_{0} V_{i j} \Psi+\frac{1}{2} G_{0}\left[V_{j k}^{(i)}+V_{k i}^{(j)}\right] \Psi .
$$

Obviously these Faddeev equations satisfy the same three-particle Hamiltonian as eq.(3), thus they provide the same solution for the systems wave function $\Psi$. Nevertheless individual Faddeev components $\widetilde{F}_{i j}^{(k)}$ differ from components $F_{i j}^{(k)}$, which satisfy eq.(3).

Instead of using eq.(2), we calculate $3 \mathrm{NF}$ expectation value using identity:

$$
\left\langle\Psi\left|V_{i j k}\right| \Psi\right\rangle=\frac{3}{2}\left\langle\Psi\left|V_{j k}^{(i)}+V_{k i}^{(j)}\right| \Psi\right\rangle .
$$

Now eq.(7) is replaced with:

$$
\Psi^{\prime} \sim \frac{1}{2}\left[W^{(k)} I^{(j)} W^{(i)}+W^{(k)} I^{(i)} W^{(j)}\right] \Psi .
$$

Sum of first two terms $\left(C_{1}\right.$ and $C_{3}$ ones) of 2-pion exchange force in eq.(6) we propose to evaluate as:

$$
\begin{aligned}
{ }_{k}\left\langle p^{\prime \prime \prime} q^{\prime \prime \prime} \alpha^{\prime \prime \prime} \mid \Psi^{\prime}\right\rangle= & \frac{1}{2} \sum^{\prime \prime} \sum^{\prime} \frac{(-)^{J-J^{\prime}}}{\widehat{J}} \\
& \times\left({ }_{k}\left\langle p^{\prime \prime \prime} q^{\prime \prime \prime} \alpha^{\prime \prime \prime}\left\|W^{(k)}\right\| p^{\prime \prime} q^{\prime \prime} \alpha^{\prime \prime}\right\rangle_{k}{ }_{k}\left\langle p^{\prime \prime} q^{\prime \prime} \alpha^{\prime \prime}\left|I^{(j)}\right| p^{\prime} q^{\prime} \alpha^{\prime}\right\rangle_{i}\right. \\
& \times{ }_{i}\left\langle p^{\prime} q^{\prime} \alpha^{\prime}\left\|W^{(i)}\right\| p q \alpha\right\rangle_{i}{ }_{i}\langle p q \alpha \mid \Psi\rangle \\
& +{ }_{k}\left\langle p^{\prime \prime \prime} q^{\prime \prime \prime} \alpha^{\prime \prime \prime}\left\|W^{(k)}\right\| p^{\prime \prime} q^{\prime \prime} \alpha^{\prime \prime}\right\rangle_{k}{ }_{k}\left\langle p^{\prime \prime} q^{\prime \prime} \alpha^{\prime \prime}\left|I^{(i)}\right| p^{\prime} q^{\prime} \alpha^{\prime}\right\rangle_{j} \\
& \left.\times{ }_{j}\left\langle p^{\prime} q^{\prime} \alpha^{\prime}\left\|W^{(j)}\right\| p q \alpha\right\rangle_{j}{ }_{j}\langle p q \alpha \mid \Psi\rangle\right) .
\end{aligned}
$$

Since systems wave function is completely antisymmetric, one has ${ }_{i}\langle p q \alpha \mid \Psi\rangle \equiv_{j}\langle p q \alpha \mid \Psi\rangle$. Furthermore $W\left(\vec{Q}_{j}\right)$ is symmetric for exchange of the particles $k$ and $i$, thus product ${ }_{j}\left\langle p^{\prime} q^{\prime} \alpha^{\prime}\left\|W^{(j)}\right\| p q \alpha\right\rangle_{j}\langle p q \alpha \mid \Psi\rangle$ is a vector antisymmetric for exchange of the particles $k$ and $i$. One can further demonstrate simple relation between matrix elements of ${ }_{k}\left\langle p^{\prime \prime} q^{\prime \prime} \alpha^{\prime \prime}\left|I^{(j)}\right| p^{\prime} q^{\prime} \alpha^{\prime}\right\rangle_{i}$ and ${ }_{k}\left\langle p^{\prime \prime} q^{\prime \prime} \alpha^{\prime \prime}\left|I^{(i)}\right| p^{\prime} q^{\prime} \alpha^{\prime}\right\rangle_{j}$ :

$$
{ }_{k}\left\langle p^{\prime \prime} q^{\prime \prime} \alpha^{\prime \prime}\left|I^{(j)}\right| p^{\prime} q^{\prime} \alpha^{\prime}\right\rangle_{i}=(-)^{t_{p}^{\prime \prime}+s_{p}^{\prime \prime}+l_{p}^{\prime \prime}}(-)^{t_{p}^{\prime}+s_{p}^{\prime}+l_{p}^{\prime}}{ }_{k}\left\langle p^{\prime \prime} q^{\prime \prime} \alpha^{\prime \prime}\left|I^{(i)}\right| p^{\prime} q^{\prime} \alpha^{\prime}\right\rangle_{j},
$$

where two phase factors reflect the symmetry of bra and ket states with respect to exchange of two active particles. Second phase factor is negative, since ket state is antisymmetric for (ki) pair. Furthermore matrices ${ }_{i}\left\langle p^{\prime} q^{\prime} \alpha^{\prime}\left|W^{(i)}\right| p q \alpha\right\rangle_{i}$ and ${ }_{j}\left\langle p^{\prime} q^{\prime} \alpha^{\prime}\left|W^{(j)}\right| p q \alpha\right\rangle_{j}$ are identical. Then it is easy to see that two terms in parentheses of eq.(12) cancel each other, unless the $\left|p^{\prime \prime} q^{\prime \prime} \alpha^{\prime \prime}\right\rangle_{k}$ states are antisymmetric for exchange of the particles $i$ and $j$ (as should be, since $V_{j k}^{(i)}+V_{k i}^{(j)}$ as well as $W^{(k)}$ are symmetric in $(i j)$ pair). When $\left|p^{\prime \prime} q^{\prime \prime} \alpha^{\prime \prime}\right\rangle_{k}$ are physical, antisymmetric for exchange of the particles $i$ and $j$, two terms in eq.(12) give identical contributions. Finally, one can write:

$$
\begin{aligned}
{ }_{k}\left\langle p^{\prime \prime \prime} q^{\prime \prime \prime} \alpha^{\prime \prime \prime} \mid \Psi^{\prime}\right\rangle= & \sum^{\prime \prime} \sum^{\prime} \frac{(-)^{J-J^{\prime}}}{\widehat{J}}{ }_{k}\left\langle p^{\prime \prime \prime} q^{\prime \prime \prime} \alpha^{\prime \prime \prime}\left\|W^{(k)}\right\| p^{\prime \prime} q^{\prime \prime} \alpha^{\prime \prime}\right\rangle_{k} \\
& \times{ }_{k}\left\langle p^{\prime \prime} q^{\prime \prime} \alpha^{\prime \prime}\left|I^{(i)}\right| p^{\prime} q^{\prime} \alpha^{\prime}\right\rangle_{j}{ }_{j}\left\langle p^{\prime} q^{\prime} \alpha^{\prime}\left\|W^{(j)}\right\| p q \alpha\right\rangle_{j}{ }_{j}\langle p q \alpha \mid \Psi\rangle
\end{aligned}
$$

This expression contains only one particle-base transformation operation, which acts only between the physical states (ones being antisymmetric with respect to active particle pair). On the contrary old PWD eq. (8) required two particlebase transformation operations, which were coupling both unphysical (symmetric) and physical (antisymmetric) states. 
Now we turn our attention to the $C_{4}$ term of eq.([6), let evaluate it according to:

$$
\begin{aligned}
{ }_{k}\left\langle p^{\prime \prime \prime} q^{\prime \prime \prime} \alpha^{\prime \prime \prime} \mid \Psi^{\prime}\right\rangle \sim & \frac{1}{2} \sum^{\prime \prime} \sum^{\prime} \frac{(-)^{J-J^{\prime}}}{\widehat{J}} \\
& \left({ }_{k}\left\langle p^{\prime \prime \prime} q^{\prime \prime \prime} \alpha^{\prime \prime \prime}\left\|\vec{W}_{b}^{(k)}\right\| p^{\prime \prime} q^{\prime \prime} \alpha^{\prime \prime}\right\rangle_{k}{ }_{k}\left\langle p^{\prime \prime} q^{\prime \prime} \alpha^{\prime \prime}\left|I_{0}\right| p^{\prime} q^{\prime} \alpha^{\prime}\right\rangle_{i}\right. \\
& \times{ }_{i}\left\langle p^{\prime} q^{\prime} \alpha^{\prime}\left\|^{(j)} \vec{W}_{d}^{(i)}\right\| p q \alpha\right\rangle_{i}{ }_{i}\langle p q \alpha \mid \Psi\rangle \\
& +{ }_{k}\left\langle p^{\prime \prime \prime} q^{\prime \prime \prime} \alpha^{\prime \prime \prime}\left\|\vec{W}_{b}^{(k)}\right\| p^{\prime \prime} q^{\prime \prime} \alpha^{\prime \prime}\right\rangle_{k}{ }_{k}\left\langle p^{\prime \prime} q^{\prime \prime} \alpha^{\prime \prime}\left|-I_{0}\right| p^{\prime} q^{\prime} \alpha^{\prime}\right\rangle_{j} \\
& \left.\times{ }_{j}\left\langle p^{\prime} q^{\prime} \alpha^{\prime}\left\|^{(i)} \vec{W}_{d}^{(j)}\right\| p q \alpha\right\rangle_{j}{ }_{j}\langle p q \alpha \mid \Psi\rangle\right) .
\end{aligned}
$$

One should note the minus sign in front of the isospin term in the second line, which appears when splitting $V_{j k}^{(i)}$ into the product $I_{0}\left(\vec{W}_{b}^{(k)} \cdot{ }^{(i)} \vec{W}_{d}^{(j)}\right)$. The matrix elements of the potentials ${ }^{(j)} \vec{W}_{d}^{(i)}$ and ${ }^{(i)} \vec{W}_{d}^{(j)}$ are equal up to phase factor:

$$
{ }_{i}\left\langle p^{\prime} q^{\prime} \alpha^{\prime}\left\|^{(j)} \vec{W}_{d}^{(i)}\right\| p q \alpha\right\rangle_{i}=(-)^{s_{p}^{\prime}-s_{p}}{ }_{j}\left\langle p^{\prime} q^{\prime} \alpha^{\prime}\left\|^{(i)} \vec{W}_{d}^{(j)}\right\| p q \alpha\right\rangle_{j}
$$

Term ${ }^{(i)} \vec{W}_{d}^{(j)}$ is not anymore symmetric with respect to exchange of the particles $k$ and $i$, therefore these matrices also couple physical (antisymmetric) states with the unphysical (symmetric) ones. Basically, phase factor in eq. (16) is positive for the transition between the physical states and is negative when one pass from physical state to an unphysical one. For the matrix elements of the isospin operators one has relation:

$$
{ }_{k}\left\langle p^{\prime \prime} q^{\prime \prime} \alpha^{\prime \prime}\left|I_{0}\right| p^{\prime} q^{\prime} \alpha^{\prime}\right\rangle_{i}=(-)^{t_{p}^{\prime \prime}+s_{p}^{\prime \prime}+l_{p}^{\prime \prime}}(-)^{t_{p}^{\prime}+s_{p}^{\prime}+l_{p}^{\prime}}{ }_{k}\left\langle p^{\prime \prime} q^{\prime \prime} \alpha^{\prime \prime}\left|-I_{0}\right| p^{\prime} q^{\prime} \alpha^{\prime}\right\rangle_{j}
$$

Since sum of potential's terms $V_{j k}^{(i)}+V_{k i}^{(j)}$ as well as term $\vec{W}_{b}^{(k)}$ are symmetric with respect to exchange of the particles $i$ and $j$ the state $\left|p^{\prime \prime} q^{\prime \prime} \alpha^{\prime \prime}\right\rangle_{k}$ present in the eq.(15) is antisymmetric for $(i j)$ pair: thus first phase factor in eq.(17) is negative. Overall phase factor is negative if $\left|p^{\prime} q^{\prime} \alpha^{\prime}\right\rangle_{j}$ state is symmetric, whereas overall phase factor is positive if this state is antisymmetric with respect to pair $(k i)$. Summarizing these remarks one can see that two additive terms in eq.(15) are identical, giving the matrix expression:

$$
\begin{aligned}
{ }_{k}\left\langle p^{\prime \prime \prime} q^{\prime \prime \prime} \alpha^{\prime \prime \prime} \mid \Psi^{\prime}\right\rangle= & -\sum^{\prime \prime} \sum^{\prime} \frac{(-)^{J-J^{\prime}}}{\widehat{J}}{ }_{k}\left\langle p^{\prime \prime \prime} q^{\prime \prime \prime} \alpha^{\prime \prime \prime}\left\|\vec{W}_{b}^{(k)}\right\| p^{\prime \prime} q^{\prime \prime} \alpha^{\prime \prime}\right\rangle_{k} \\
& \times{ }_{k}\left\langle p^{\prime \prime} q^{\prime \prime} \alpha^{\prime \prime}\left|I_{0}\right| p^{\prime} q^{\prime} \alpha^{\prime}\right\rangle_{j}{ }_{j}\left\langle p^{\prime} q^{\prime} \alpha^{\prime}\left\|^{(i)} \vec{W}_{d}^{(j)}\right\| p q \alpha\right\rangle_{j}{ }_{j}\langle p q \alpha \mid \Psi\rangle
\end{aligned}
$$

This time $\left|p^{\prime} q^{\prime} \alpha^{\prime}\right\rangle_{j}$ basis contain both physical and unphysical states, nevertheless other states $\left(\alpha, \alpha^{\prime \prime}\right.$ and $\left.\alpha^{\prime \prime \prime}\right)$ are physical.

Nevertheless one can avoid arithmetics with unphysical states even evaluating term $C_{4}$. In that aim we use identity $\vec{\sigma}_{k}=2 \vec{S}-\vec{\sigma}_{i}-\vec{\sigma}_{j}$ with $\vec{S}$ representing total spin of three nucleon system to rewrite $C_{4}$ term:

$$
C_{4}\left({ }^{(k)} \vec{W}_{d}^{(j)} \cdot \vec{W}_{b}^{(i)}\right) I_{0}=C_{4}\left(\vec{W}_{e}^{(j)} \cdot \vec{W}_{b}^{(i)}\right) I_{0}+C_{4}\left(\vec{W}_{b}^{(j)} \cdot \vec{W}_{f}^{(i)}\right) I_{0},
$$

where $\vec{W}_{e}^{(j)}=\tilde{f}_{j}\left(\vec{\sigma}_{j} \cdot \vec{Q}_{j}\right)\left[\vec{Q}_{j} \times\left(2 \vec{S}_{-} \vec{\sigma}_{j}\right)\right]$ and $\vec{W}_{f}^{(i)}=\tilde{f}_{i}\left(\vec{\sigma}_{i} \cdot \vec{Q}_{i}\right)\left[\vec{Q}_{i} \times \vec{\sigma}_{i}\right]$. The particular form of the last operator, as it is easily demonstrated in the Appendix, results in $\vec{W}_{f}^{(i)} \equiv 0$. One can see that the term $\vec{W}_{e}^{(j)}$ is symmetric for nucleon pair $(k i)$. Therefore arguments used to derive eq.(14) can be followed to demonstrate:

$$
\begin{aligned}
{ }_{k}\left\langle p^{\prime \prime \prime} q^{\prime \prime \prime} \alpha^{\prime \prime \prime} \mid \Psi^{\prime}\right\rangle= & -\sum^{\prime \prime} \sum^{\prime} \frac{(-)^{J-J^{\prime}}}{\widehat{J}}\left({ }_{k}\left\langle p^{\prime \prime \prime} q^{\prime \prime \prime} \alpha^{\prime \prime \prime}\left\|\vec{W}_{b}^{(k)}\right\| p^{\prime \prime} q^{\prime \prime} \alpha^{\prime \prime}\right\rangle_{k}\right. \\
& \left.\times{ }_{k}\left\langle p^{\prime \prime} q^{\prime \prime} \alpha^{\prime \prime}\left|I_{0}\right| p^{\prime} q^{\prime} \alpha^{\prime}\right\rangle_{j}{ }_{j}\left\langle p^{\prime} q^{\prime} \alpha^{\prime}\left\|\vec{W}_{e}^{(j)}\right\| p q \alpha\right\rangle_{j}{ }_{j}\langle p q \alpha \mid \Psi\rangle\right)
\end{aligned}
$$

This expression comprise only physical states and requires single basis transformation operation. Evaluation of $3 \mathrm{NF}$ terms using old PWD of eq.(8) required two basis transformation operations: one ${ }_{j}\left\langle p^{\prime \prime} q^{\prime \prime} \alpha^{\prime \prime}|I| p^{\prime} q^{\prime} \alpha^{\prime}\right\rangle_{i}$ involving unphysical and physical states in both ket and bra parts (this transformation is numerically at least four times heavier than one coupling only physical states), whereas other ${ }_{k}\left\langle p^{\prime \prime \prime \prime} q^{\prime \prime \prime \prime} \alpha^{\prime \prime \prime \prime} \mid p^{\prime \prime \prime} q^{\prime \prime \prime} \alpha^{\prime \prime \prime}\right\rangle_{j}$ transforming combination of 
unphysical and physical ket states into the physical bra state (this transformation is numerically at least two times heavier than one coupling only physical states). As mentioned in [1] the basis transformation operations consumes essentially all the CPU time required to evaluate $3 \mathrm{NF}$ terms, while the time for the calculation of ${ }_{i}\left\langle p^{\prime} q^{\prime} \alpha^{\prime}\left\|W^{(i)}\right\| p q \alpha\right\rangle_{i}$ matrix elements is negligible. Therefore two-pion exchange $3 \mathrm{NF}$ terms can be evaluated by as much as six times faster using eq.(14) and eq.(20) than using old PWD.

In the end I should remark that presented method can be applied to evaluate any momentum or coordinate space $3 \mathrm{NF}$, which has a form: $V_{i j k}=\sum_{c y c} V_{i j} V_{j k}$. Application of similar PWD technique to evaluate UIX $3 \mathrm{NF}$ has been demonstrated in [6].

It is clear that described method is not directly applicable for more complex $3 \mathrm{NF}$, which involves more than two meson exchanges, like Illinois force containing three-pion ring terms [8]. Nevertheless variation of this technique is possible to evaluate also the terms of type $V_{i j k}=\sum_{c y c} V_{i j} V_{j k} W_{k i}$ without introducing mixing with unphysical states.

\section{APPENDIX A:}

Here we give explicit formulaes for the potential's terms $W$, required to evaluate two-pion exchange 3 NF. When working with Faddeev equations one deals with binary particle interactions, which in proper Jacobi coordinate set are expressed as the functions of momenta $p$ (and/or $p^{\prime}$ ) and which are independent of momenta $q\left(q^{\prime}\right)$. In contrary, our expressions for $W^{(j)}$-terms are the functions of $\vec{Q}_{j}=\vec{k}_{j}^{\prime}-\vec{k}_{j} \equiv \vec{q}_{j}^{\prime}-\vec{q}_{j}$ and they are diagonal in Jacobi momentum $p_{j}$. In this sense evaluation of $W$-terms are different from the evaluation of two-nucleon interaction terms, still they are similar in numerical complexity.

The scalar potential term $W_{a}^{(j)}=\widetilde{f}_{j}\left(\vec{\sigma}_{j} \cdot \vec{Q}_{j}\right)$ is the simplest case to evaluate:

$$
\begin{aligned}
j\left\langle p^{\prime} q^{\prime} \alpha^{\prime}\left|W_{a}^{(j)}\right| p q \alpha\right\rangle_{j}= & \frac{\delta\left(p^{\prime}-p\right)}{p^{\prime} p} \delta_{l_{p}} l_{p}^{\prime} \delta_{j_{p} j_{p}^{\prime}} \delta_{s_{p} s_{p}^{\prime}} \delta_{t_{p} t_{p}^{\prime}} \delta_{j_{q} j_{q}^{\prime}} \delta_{J J^{\prime}} \delta_{M M^{\prime}} \delta_{T T^{\prime}} 6 \pi \sqrt{2 \widehat{l}_{q} \widehat{l}_{q}^{\prime}}(-)^{j_{q}+l_{q}+\frac{3}{2}} \\
& \times\left\{\begin{array}{ccc}
l_{q}^{\prime} & l_{q} & 1 \\
\frac{1}{2} & \frac{1}{2} & j_{q}
\end{array}\right\} \sum_{\lambda} \sum_{k_{1}+k_{2}=1} \hat{\lambda}\left(\begin{array}{ccc}
k_{1} & \lambda & l_{q}^{\prime} \\
0 & 0 & 0
\end{array}\right)\left(\begin{array}{ccc}
k_{2} & \lambda & l_{q} \\
0 & 0 & 0
\end{array}\right) \\
& \times\left\{\begin{array}{ccc}
k_{2} & l_{q} & \lambda \\
l_{q}^{\prime} & k_{1} & 1
\end{array}\right\} q^{\prime k_{1}} q^{k_{2}} g_{\lambda 2},
\end{aligned}
$$

with

$$
g_{\lambda K}=\int_{-1}^{1} d u P_{\lambda}(u) \frac{f(\widetilde{Q})}{Q^{2}+M_{\pi}^{2}} \frac{Q^{2}}{Q^{K}} .
$$

In the last expression $Q=\sqrt{q^{2}+q^{\prime 2}-2 q q^{\prime} u}, P_{\lambda}(u)$ is Legendre polynomial. The $f(\widetilde{Q})$ is model defined nucleon high-momentum cut-off function as introduced in eq.(15): in some models, as [4, 5], cut-off is set on momentum transfer $\widetilde{Q} \equiv Q$; in other models, as [3], cut-off can be set on single particle momenta $\widetilde{Q} \equiv\left(q, q^{\prime}\right)$. One should note that the operator $W_{a}^{(j)}$ conserves the three-nucleon angular momentum $J$, nevertheless it changes the parity of the state to its opposite.

Next step is to evaluate reduced matrix elements of the vector potential $\vec{W}_{b}^{(j)}=\widetilde{f}_{j}\left(\vec{\sigma}_{j} \cdot \vec{q}_{j}\right) \vec{q}_{j}$ :

$$
\begin{aligned}
& j_{j}\left\langle p^{\prime} q^{\prime} \alpha^{\prime}\left\|\vec{W}_{b}^{(j)}\right\| p q \alpha\right\rangle_{j}=\frac{\delta\left(p^{\prime}-p\right)}{p^{\prime} p} \delta_{l_{p} l_{p}^{\prime}} \delta_{j_{p} j_{p}^{\prime}} \delta_{s_{p} s_{p}^{\prime}} \delta_{t_{p} t_{p}^{\prime}} \delta_{T T^{\prime}} 2 \pi \sum_{\substack{\lambda \\
k_{1}+k_{2}=K \\
K=0,2}} \widehat{\lambda} \widehat{K} \\
& \times\left(1+k_{1} k_{2}\right) \sqrt{\frac{k_{1} ! k_{2} !}{\widehat{k}_{1} ! ! \widehat{k}_{2} ! !}} \sqrt{6 \widehat{k}_{1} \widehat{k}_{2} \widehat{l}_{q} \widehat{l}_{q}^{\prime} \widehat{j}_{q} \widehat{j}_{q}^{\prime} \widehat{J} \widehat{J}^{\prime}}(-)^{k_{2}+J^{\prime}+j_{q}+j_{p}} \\
& \times\left(\begin{array}{ccc}
k_{1} & \lambda & l_{q}^{\prime} \\
0 & 0 & 0
\end{array}\right)\left(\begin{array}{ccc}
k_{2} & \lambda & l_{q} \\
0 & 0 & 0
\end{array}\right)\left\{\begin{array}{ccc}
j_{q} & j_{p} & J \\
J^{\prime} & 1 & j_{q}^{\prime}
\end{array}\right\}\left\{\begin{array}{ccc}
k_{2} & l_{q} & \lambda \\
l_{q}^{\prime} & k_{1} & K
\end{array}\right\} \\
& \times\left\{\begin{array}{ccc}
K & 1 & 1 \\
l_{q}^{\prime} & \frac{1}{2} & j_{q}^{\prime} \\
l_{q} & \frac{1}{2} & j_{q}
\end{array}\right\} q^{\prime k_{1}} q^{k_{2}} g_{\lambda K}
\end{aligned}
$$


Finally, we present expressions for the reduced matrix elements of the vector potentials ${ }^{(k)} \vec{W}_{d}^{(j)}=$ $\tilde{f}_{i}\left(\vec{\sigma}_{j} \cdot \vec{Q}_{j}\right)\left[\vec{Q}_{j} \times \vec{\sigma}_{k}\right]$ and $\vec{W}_{e}^{(j)}=\tilde{f}_{j}\left(\vec{\sigma}_{j} \cdot \vec{Q}_{j}\right)\left[\vec{Q}_{j} \times\left(2 \vec{S}-\vec{\sigma}_{j}\right)\right]:$

$$
\begin{aligned}
j_{j}\left\langle p^{\prime} q^{\prime} \alpha^{\prime}\left\|{ }^{(k)} \vec{W}_{d}^{(j)}\right\| p q \alpha\right\rangle_{j}= & \frac{\delta\left(p^{\prime}-p\right)}{p^{\prime} p} \delta_{l_{p} l_{p}^{\prime}} \delta_{t_{p} t_{p}^{\prime}} \delta_{T T^{\prime}} 12 \pi i \sum_{\lambda L L^{\prime} S S^{\prime} h \sum_{k_{1}+k_{2}=K}} \widehat{\lambda} \widehat{h} \widehat{K} \widehat{L} \widehat{L}^{\prime} \widehat{S} \widehat{S}^{\prime} \\
& \times\left(1+k_{1} k_{2}\right) \sqrt{\frac{k_{1} ! k_{2} !}{\widehat{k}_{1} ! ! \widehat{k}_{2} ! !}} \sqrt{6 \widehat{k}_{1} \widehat{k}_{2} \widehat{j}_{p} \widehat{j}_{p}^{\prime} \widehat{j}_{q} \widehat{j}_{q}^{\prime} \widehat{l}_{q} \widehat{l}_{q}^{\prime} \widehat{J} \widehat{J}^{\prime} \widehat{s}_{p} \widehat{s}_{p}^{\prime}}(-)^{L^{\prime}+k_{2}+l_{q}+l_{p}+h+1+s_{p}^{\prime}} \\
& \times\left(\begin{array}{ccc}
k_{1} & \lambda & l_{q}^{\prime} \\
0 & 0 & 0
\end{array}\right)\left(\begin{array}{ccc}
k_{2} & \lambda & l_{q} \\
0 & 0 & 0
\end{array}\right)\left\{\begin{array}{ccc}
l_{q} & l_{p} & L \\
L^{\prime} & K & l_{q}^{\prime}
\end{array}\right\}\left\{\begin{array}{ccc}
h & K & 1 \\
1 & 1 & 1
\end{array}\right\} \\
& \times\left\{\begin{array}{ccc}
k_{2} & l_{q} & \lambda \\
l_{q}^{\prime} & k_{1} & K
\end{array}\right\}\left\{\begin{array}{ccc}
\frac{1}{2} & \frac{1}{2} & s_{p}^{\prime} \\
s_{p} & 1 & \frac{1}{2}
\end{array}\right\}\left\{\begin{array}{ccc}
l_{p} & s_{p} & j_{p} \\
l_{q} & \frac{1}{2} & j_{q} \\
L & S & J
\end{array}\right\}\left\{\begin{array}{ccc}
l_{p}^{\prime} & s_{p}^{\prime} & j_{p}^{\prime} \\
l_{q}^{\prime} & \frac{1}{2} & j_{q}^{\prime} \\
L^{\prime} & S^{\prime} & J^{\prime}
\end{array}\right\} \\
& \times\left\{\begin{array}{ccc}
K & h & 1 \\
L^{\prime} & S^{\prime} & J^{\prime} \\
L & S & J
\end{array}\right\}\left\{\begin{array}{ccc}
1 & 1 & h \\
s_{p}^{\prime} & \frac{1}{2} & S^{\prime} \\
s_{p} & \frac{1}{2} & S
\end{array}\right\} q^{\prime k_{1}} q^{k_{2}} g_{\lambda K}
\end{aligned}
$$

and

$$
\begin{aligned}
j\left\langle p^{\prime} q^{\prime} \alpha^{\prime}\left\|\vec{W}_{e}^{(j)}\right\| p q \alpha\right\rangle_{j}= & \frac{\delta\left(p^{\prime}-p\right)}{p^{\prime} p} \delta_{s_{p} 1} \delta_{s_{p} s_{p}^{\prime}} \delta_{l_{p} l_{p}^{\prime}} \delta_{t_{p} t_{p}^{\prime}} \delta_{T T^{\prime}} 24 \pi i \sum_{\lambda L L^{\prime} S S^{\prime} h k_{1}+k_{2}=K} \hat{K=0,2} \text { } \widehat{h} \widehat{K} \widehat{L} \widehat{L} \widehat{L}^{\prime} \widehat{S} \widehat{S}^{\prime} \\
& \times\left(1+k_{1} k_{2}\right) \sqrt{\frac{k_{1} ! k_{2} !}{\widehat{k}_{1} ! ! \widehat{k}_{2} ! !}} \sqrt{6 \widehat{k}_{1} \widehat{k}_{2} \widehat{j}_{p} \widehat{j}_{p}^{\prime} \widehat{j}_{q} \widehat{j}_{q}^{\prime} \widehat{l}_{q} \widehat{l}_{q}^{\prime} \widehat{J} \widehat{J}^{\prime}}(-)^{L^{\prime}+k_{2}+l_{q}+l_{p}+h+1} \\
& \times\left(\begin{array}{ccc}
k_{1} & \lambda & l_{q}^{\prime} \\
0 & 0 & 0
\end{array}\right)\left(\begin{array}{ccc}
k_{2} & \lambda & l_{q} \\
0 & 0 & 0
\end{array}\right)\left\{\begin{array}{ccc}
l_{q} & l_{p} & L \\
L^{\prime} & K & l_{q}^{\prime}
\end{array}\right\}\left\{\begin{array}{ccc}
h & K & 1 \\
1 & 1 & 1
\end{array}\right\} \\
& \times\left\{\begin{array}{ccc}
k_{2} & l_{q} & \lambda \\
l_{q}^{\prime} & k_{1} & K
\end{array}\right\}\left\{\begin{array}{ccc}
l_{p} & s_{p} & j_{p} \\
l_{q} & \frac{1}{2} & j_{q} \\
L & S & J
\end{array}\right\}\left\{\begin{array}{ccc}
l_{p}^{\prime} & s_{p}^{\prime} & j_{p}^{\prime} \\
l_{q}^{\prime} & \frac{1}{2} & j_{q}^{\prime} \\
L^{\prime} & S^{\prime} & J^{\prime}
\end{array}\right\} \\
& \times\left\{\begin{array}{ccc}
K & h & 1 \\
L^{\prime} & S^{\prime} & J^{\prime} \\
L & S & J
\end{array}\right\}\left\{\begin{array}{ccc}
1 & 1 & h \\
s_{p}^{\prime} & \frac{1}{2} & S^{\prime} \\
s_{p} & \frac{1}{2} & S
\end{array}\right\} q^{\prime k_{1}} q^{k_{2}} g_{\lambda K} .
\end{aligned}
$$

The $\vec{W}_{b}^{(j)},{ }^{(k)} \vec{W}_{d}^{(j)}$ and $\vec{W}_{e}^{(j)}$ potential terms conserve the parity of three-nucleon state, however it may change its total angular momentum.

Using simple tensor algebra relations, one has:

$$
\left(\vec{\sigma}_{i} \cdot \vec{Q}_{i}\right)\left[\vec{Q}_{i} \times \vec{\sigma}_{i}\right]=3 i \sum_{g h} \sqrt{2 \widehat{g} \widehat{h}}\left\{\begin{array}{lll}
1 & 1 & 0 \\
1 & 1 & 1 \\
g & h & 1
\end{array}\right\}\left\{\left\{\vec{Q}_{i} \otimes \vec{Q}_{i}\right\}_{g} \otimes\left\{\vec{\sigma}_{i} \otimes \vec{\sigma}_{i}\right\}_{h}\right\}_{1} .
$$

In this equation the product $\left\{\vec{\sigma}_{i} \otimes \vec{\sigma}_{i}\right\}_{h}$ acts on spin one-half states and it is equal zero unless $h=0$. However $g=1$, if $h=0$ and $\left\{\vec{Q}_{i} \otimes \vec{Q}_{i}\right\}_{g=1} \equiv 0$ as a vector product of two parallel vectors vanishes. Therefore the last equation is identically zero and $\vec{W}_{f}^{(i)} \equiv 0$.

PWD basis transformation operations ${ }_{j}\left\langle p^{\prime \prime} q^{\prime \prime} \alpha^{\prime \prime}|I| p^{\prime} q^{\prime} \alpha^{\prime}\right\rangle_{i}$ have been discussed and necessary expressions have been given in ref. [1, 7]. 


\section{Acknowledgments}

I would like to thank my former Ph.D. supervisor Claude Gignoux. In fact, idea of the PWD presented in this work have born already long time ago during discussion with Claude.

[1] D. Hüber, H. Witała, A. Nogga et al., Few-Body Syst. 22 (1997) 107.

[2] AIP Conference Proceedings 1011, edited by H. Sakai, K. Sekiguchi, B.F. Gibson (AIP, 2008); http://proceedings.aip.org/dbt/dbt.jsp?KEY=APCPCS

[3] E. Epelbaum, A. Nogga, W. Glöckle et al., Phys. Rev. C66 (2002) 064001.

[4] J. L. Friar, D. Hüber, and U. van Kolck, Phys. Rev. C 59 (1999) 53.

[5] P. Navratil, Few Body Syst. 41 (2007) 117.

[6] R. Lazauskas, PhD Thesis, Université Joseph Fourier, Grenoble (2003).

[7] D. Hüber, J.L. Friar, A. Nogga et al., Few-Body Syst. 30 (2001) 95.

[8] S.C. Pieper, V.R. Pandharipande et al., Phys. Rev. C64 (2001) 014001. 